\title{
Production management: Which future?
}

\author{
Doumeingts G. , Ducq Y. \\ LAP/GRAI - Université Bordeaux I \\ 351, Cours de la Libération, 33405 Talence - FRANCE - \\ Tel : 33-(0)5 56-84-65-30 \\ Fax : 33-(0)5 56-84-66-44 - \\ Email :doumeingts@grai.lap.u-bordeaux.fr
}

\begin{abstract}
This paper discusses about the evolution of the production management in the next future. This evolution will concerns the integration of production management (PM) with the other functions inside and outside of the enterprise, the use of enterprise modelling techniques to analyse, to design and to implement production management systems with the choice of software packages. Then, we will focus on the evolution of packages with the emergence of "Integrated softwares" and the role of "object design".
\end{abstract}

\section{Keywords}

Production management, Software Packages, Integration, Enterprise modelling, Object design, Extended Enterprise.

\section{INTRODUCTION}

Due to its technical, economical social and human nature, the production management has always presented a high complexity. This complexity is also due to its particular position at the crossing of the administrative, financial, commercial, marketing, and manufacturing functions.

Moreover, the dynamic running of the PMS and the interactivity between the human organisation and the Information Technology (IT) processing make the problem solving of the PM function as one of the most difficult among the functionalities of the enterprise. This complexity increases also by the fact that there is a large variety of techniques to produce from Make To Stock (MTS) to Engineer To Order (ETO) and One of Kind Production (OKP). The obligation of cooperation between enterprises in the line of Extended Enterprise increases also the complexity of Production Management System.

Today, one very difficult task is to choose a well adapted software and to implement 
it in a short time.

Nevertheless, the evolution of the market and of the production techniques and organisations implies an evolution of the concepts of production management.

After having defined the domain and the main concepts of the production management, this paper will draw the future trends of evolution focusing on integration, use of Enterprise Modelling Techniques, and Packages Softwares.

\section{THE DOMAIN OF THE PRODUCTION MANAGEMENT AND THE SOFTWARE SUPPORTS}

After having described the various structures of Production Management, we will analyse the Software Tools which support the Production Management in the Enterprise.

\subsection{The production management function in the enterprise.}

The domain of the production management is very difficult to define. It exists a lot of definitions and depending on the point of view (managers, structure of the enterprise, type of manufacturing) the domain can change drastically.

First, we can use the traditional functions description : Planning, including Business Planning, Master Scheduling, Capacity planning, Scheduling, and the links with Material Requirement Planning, Purchasing, Procurement, and Resource management. We will see later that this description is insufficient because the dynamic behavior is not taken in account.

We can propose another point of view : a company can be decomposed in a set of functionalities organised in a network. This network is presented fig 1 (we represent only the links with production) :

In such an approach, the production management is considered as the interface between the production and the other enterprise functions. The difficulty in such a definition is that the relationships between the functions are conflicting. So, the production management definition depends on the point of view taken in consideration. This approach is difficult to implement due to the various models which could be deduced.

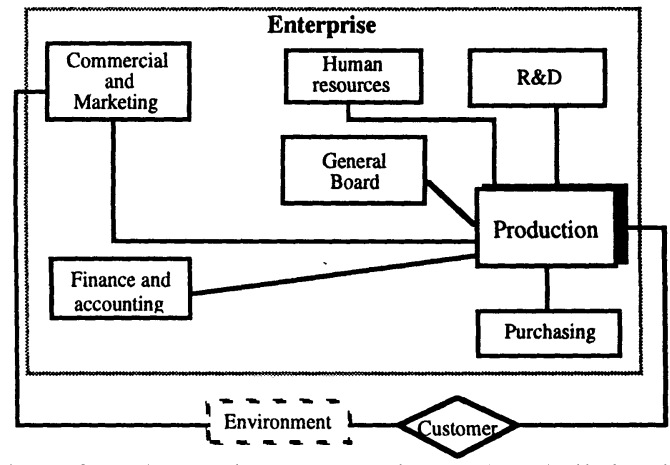

Figure 1 The various functions of the enterprise and their link with the production function. 
As an example of the description of the production system based on functions, there is the architecture developed by IMPACS Project (Integrated Manufacturing Planning And Control System) (figure 2).

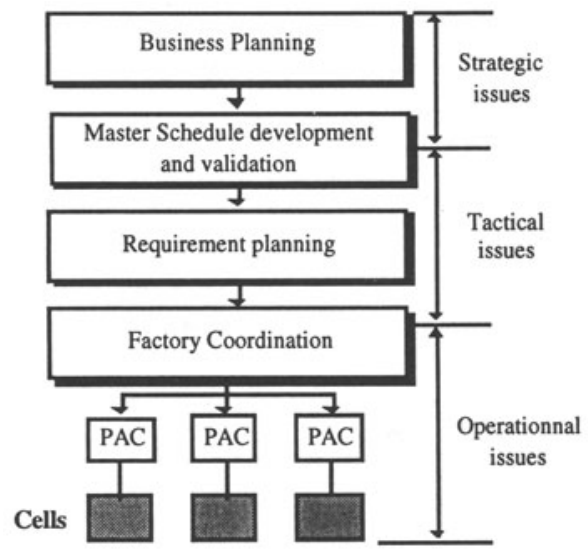

Figure 2 The CIMRU Architecture for Production Management System.

This architecture has two parts : the upper part which presents a classical decomposition, a lower part which depends on the possibilities to decompose the physical system (shopfloor) in cells (using for example Group Technology). Anyway, the trends, today, is to decompose the workshop in cells (or sections) well defined. In such a way, we can apply the concepts of PAC (Production Activity Control).

At the business planning stage, emphasis is placed on planning in terms of aggregated product families or modules. The master scheduling phase attempts to plan end level items, frequently but not always, product which are shipped to the customer. Requirements Planning is concerned with translating the master schedule items into components requirements for short term planning and purchasing. Factory coordination and Production Activity Control mainly deal with the short term (manufacturing items and assembly products).

We can also use a third approach to define production management : the systemic approach. In the systemic, three points of view are required to define a system : a functional one, a structural one and a dynamic one.

For the functional point of view, we can use the previous description particularly the IMPACS 's proposition.

For the structural view, we describe the components of the Production System (products, machines, workstations, routes ...).

For the dynamic view, we take in consideration the control of the Production System : this control must allow to reach the main objectives which deal with the optimisation of the triplet : quality, cost and time.

In such way, the systemic approach proposes to decompose the System in two sub systems : the controlled system that we will call the "Physical System" and the control system which is in our point of view the "Production Management".

This control system will be decomposed again into two sub-systems : the decision and 
the information.

The first one aims at elaborating the various decisions transmitted to the physical system. The second one allows to transmit, to process and to record the required information. It is the link between the physical and the decision sub-systems.

The decision and the information sub-systems are strong connected. So, a good synchronisation between both contributes to a satisfying running of the production management system.

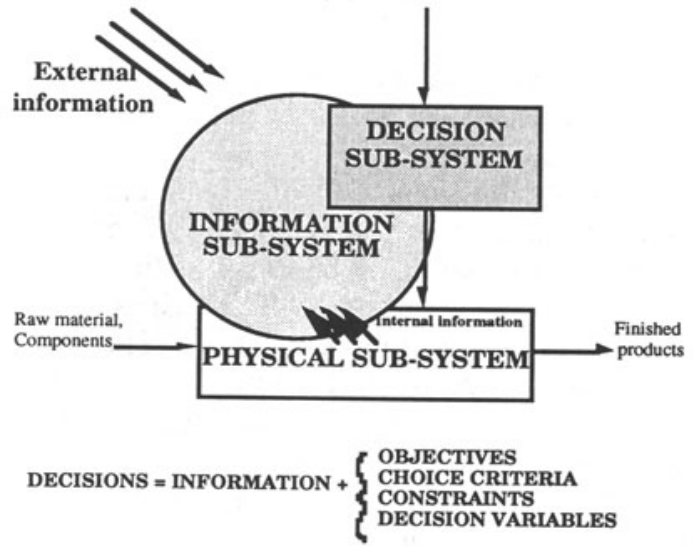

Figure 3 The systemic approach of the production system

One of our conclusions is the lack of attention given by the specialists to the model of production management. There is too much attention given to the functional view, but it is not sufficient. It is necessary to be more precise and to not forget the systemic approach including the Business Process approach.

\subsection{The software tools to support the production management.}

Today, in a modern manufacturing system, the use of software tools is necessary. At the beginning of the seventies, the important companies have developed their own softwares. It was the case till the middle of the eighties. The situation is changing now : the important firms buy software packages from the market as well as the SME's in order to improve their competitiveness. The evolution of the software packages was very slow till the end of the eighties. The structures of such softwares were quite identical, and it was very difficult to adapt these packages to the various characteristics of the manufacturing systems.

The most difficult in the acquisition phase of the software package is the choice. It existed on the market an impressive list of packages but unfortunately, only perhaps 20 of them give satisfaction on operational point of view.

The second problem to face, was the choice among the list of the most adapted packages to the characteristics of the manufacturing system. We were always surprised in the expression CAPM (Computer Aided Production Management) by the interest on "Computer Aided" (analysis of the majority of existing packages by the potential customers) and the poor interest on the "Production Management" model. Often, the specifications were not elaborated seriously and the choice was more an hazardous game than a serious evaluation of the candidate packages. 
We must recognise also that in this recent past, the majority of Software Packages were MRP II type. Few Packages were developed for the other types of manufacturing : process, one-of-a-kind... These were more "home made softwares". But now, the situation is changing.

\section{EVOLUTION OF THE FUNCTIONS}

The search of a multi-criteria performance for the production system requires for the company to be customer oriented, to anticipate the customer needs and to adapt its structure to the market changes.

The integration of the various functions of the production management system allows to answer to this situation with ensuring not only the convergence of the objectives of the functions but also the convergences of the actions. This integration is based on the capacity of cooperation inside the organisation. The various tasks are not decomposed and the functions are not compartmentalised any more. The only optimum is the one of the enterprise rather that the one of each function. To be efficient, the integration requires an organisation which defines the objectives, organises the cooperations, circulates information, dispatches and coordinates responsibilities.

Moreover, due to the rapid changes of the market, the enterprise must be reactive. This reactivity to the changes must involve the totality of the production functions. So, this reactivity is increased if these functions are running together in the same time toward the same goal, it means are coordinated. This is allowed also by the integration of the functions.

However, this integration does not imply the centralisation of the decisions and of the responsibilities but on the contrary the decentralisation of these decisions by the decentralisation of the responsibilities, allowing to react as early as possible and to increase the people motivation.

This motivation and this integration are finally also required to facilitate the evolution of the production management.

Indeed, the integration allows to manage coherently several parallel projects which aim at changing progressively the structure of the production system with the minimum of disturbances. Moreover, the motivation of employees due to the decentralisation is essential in the evolution process because it ensure the appropriation of the new system by its main actors. It also avoids to build a system too far from the expectations of the employees.

In the production system, this integration is present at several levels. First, this integration must be effective between the production function and the other enterprise functions : R\&D, Accounting, Marketing, .... In a second time, the various production functions must be integrated : design, engineering, manufacturing, delivery, and even recycling.

In the production management domain, this integration become also stronger and stronger between the various PM functions : to manage product, to manage resources, to plan , To control quality, To manage maintenance...

The definition of the quality control is to ensure that the product meets the customer requirements.

In the same time, the maintenance function contributes to the decreasing of the disturbances. 
We must mention the distribution of products (with all the logistic problems) and the relations with the suppliers (Supply Chain). This new situation increases the complexity of the planning system which is no more an internal function but must be also combined with the planning of the partners in a distributed way.

The emergence of the Extended Enterprise and the Virtual Enterprise, where separate companies agree to work together across the value chain to meet particular markets needs, will only strengthen the trend towards integrated logistic systems.

A further complication is the increasing on environmentally benign production and the emerging interest in products takeback and recovery at the end of life cycle. This requires the development of information systems and bills of material to support product disassembly, module and components recovery for reuse and ultimately material recycling.

In such a description, we must mention the use of Business Process modelling techniques : The Business Process describe a chain of interrelated activities which normally must be connected with the customer requirements. In such a description, the Porter Value Chain help the company to define the most interesting activities for the company (an economic but also technical and organisational point of view). This analysis will allow in particular to determine the configuration of the Supply Chain.

\section{THE ROLE OF ENTERPRISE MODELLING TECHNIQUES IN PRODUCTION MANAGEMENT}

The Enterprise Modelling Techniques (EMT) allows the representation of the manufacturing system thanks to :

- a conceptual model defining the various concepts, the functionalities, the structures and the dynamic behaviuor (see 2.2),

- formalisms allowing to describe and to represent this conceptual model,

- a structured approach guiding the use of the method in order to elaborate the PM model.

Indeed, the complexity of the current production management systems leads, today, to use such techniques to understand, to analyse, to design and then to improve the system.

In order to perform the modelling, a huge amount of information must be gathered and structured. These information come from many disciplines and are disseminated among various users. Moreover, there is a need to integrate economic, human and technical aspects.

It is obvious that to overcome these difficulties, an EMT is required. Moreover, the efficiency of the production management system is required both in terms of cost and time. So, its benefits can be expressed not only on the technical point of view in facilitating the achievement of relevant solutions but also on the financial point of view with decreasing the overcost due to the design mistakes.

The clear and precise representation of the system with the EMT allows to facilitate the understanding of the system running by the users, and then to ensure continuously their involvement in the change processes.

The specifications of the future system would be used not only for the design but also for the choice of a CAPM system, the definition of its parameters, and for its implementation : wrong specifications could lead to a disaster which would be discovered during the running. So, thanks to the EMT, the production system is 
improved before its computerisation.

The modelling of Production System by EMT could facilitate the implementation of the chosen Package, allowing a step by step implementation. With EMT, it is also possible to elaborate the IT Model of the PM Package, to compare with the complete model of the dedicated PM and to determine the adjustments on the both sides.

We think it is very important to be able to determine the difference between the both structure (PM and Package) before to start the implementation.

The EMT improve the running with supplying documentation.

Finally an EMT can allow to design and to implement a Performance Indicator System (it is the case of GIM [Grai Integrated Methodology] with ECOGRAI method) which aims at measuring the performance of the new system according to its objectives.

To illustrate this paragraph, one can cite the three EMT accepted by the Task Force IFAC/IFIP : PERA, CIM-OSA and GIM.

- PERA was developed at the Purdue Laboratory for Applied Industrial Control of the PURDUE University. PERA is aiming at describing the life cycle of a CIM project from the initial concepts through a functional analysis, a functional design (or specifications), a detailed design, its development and its implementation until its recycling.

PERA highlights the need to consider the human role in a CIM project. In the other side, it does not use modelling formalisms but justifies the need for a structured approach.

- The Open System Architecture for Computer Integrated Manufacturing was developed by the European CIM Architecture (AMICE) consortium under ESPRIT projects 688, 2422 and 5288 of the European Community. The objectives of CIMOSA is to develop a CIM reference architecture. The two main results of the CIMOSA project are a modelling framework for CIM and an integrated infrastructure. The modelling framework for CIM supports all phases of a CIM system life cycle from requirements definition, through design specification, implementation description and execution of the daily enterprise operation. The CIM-OSA integrating infrastructure provides specific information technology services for the execution of the particular implementation model, but, more important for vendors, it provides independence and portability.

- GIM was developed at the GRAI Laboratory and particularly through the ESPRIT projects 418 OCS, IMPACS (Integrated Manufacturing Planning And Control System) and FLEXQUAR 6408 . GIM is based on the GRAI model.

The GRAI model proposes a hierarchical and decentralised decomposition of the production system. At the physical level, the system is decomposed in shops and then in cells, according to criteria which can be technical, organisational, or social. At the decisional level, the decomposition criteria allow to determine the decision making levels and, at each level, the various decision centres (figure 4).

To describe this model, GIM uses three types of formalisms. IDEF0 modelling technique plus other techniques including the simulation for the physical system modelling, entity-relationships formalisms for the informational system and GRAI grid and GRAI nets for the decisional system. The GRAI model allows to take into account the integration features. To build the various models, GIM uses a structured approach.

One of the particularities of GIM is to consider two design domains (to build the new 
manufacturing system):

- the user oriented domain which defines the user oriented specifications through four models (functional, decisional, informational, physical),

- the technical oriented domain which defines the technical specifications divided in three parts (organisation, information technology, manufacturing technology).

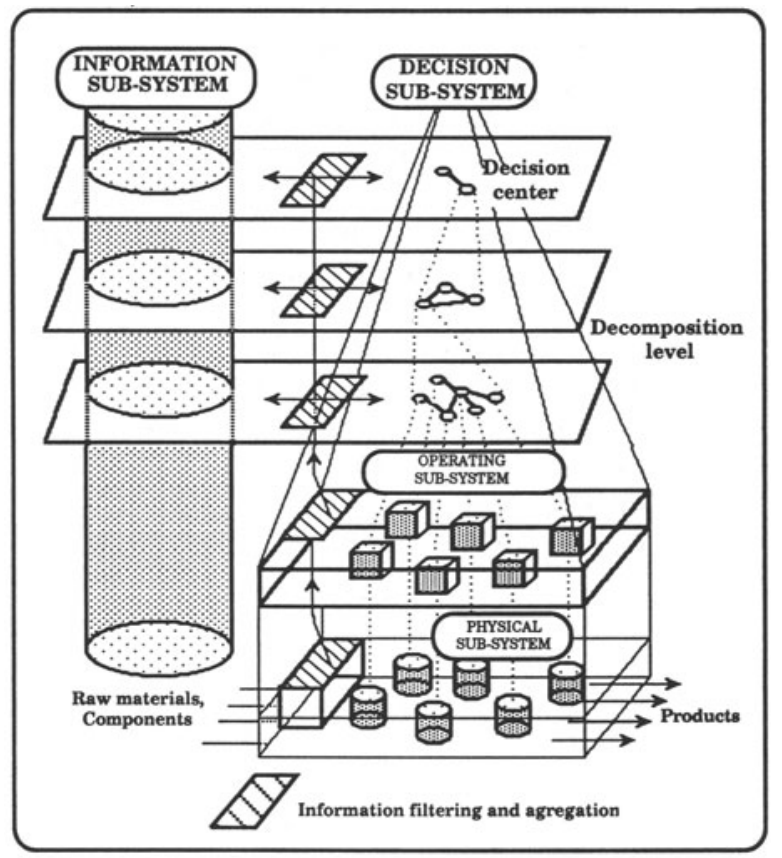

Figure 4 The GRAI model

It exists also other methodology which facilitate the implementation of Production Management Packages as ARIS developed by Prof. SCHEER (university of Saarbrucken), Procedure Analysis and Design (Mega International), METIS (NCR, Norvege)

In conclusion, it become obvious that the EMT will take a preponderant part in the Production Management with supporting BPR activities and facilitating the implementation of PM Packages.

The Modelling of PMS by EMT can allow to use the simulation techniques in order to anticipate on the evolution of Production System. More and more, in order to be reactive, it could be important to simulate the evolution according to various hypothesis.

\section{EVOLUTION OF THE PACKAGES FOR PRODUCTION MANAGEMENT}

The increasing of complexity of production management and the current progresses of softwares and hardwares led to a necessary improvement of the packages for production management. The main recent improvements are the following. 


\subsection{The development of client/server systems and the integration.}

At the end of the eighties, enterprises realised the necessity of independence with regard to the hardware and software solutions. So, there is an emergence of the concept of "Open Architectures and Systems", these systems requiring communication links.

Moreover, the delocalisation of decision centres due to the integration of the functions, as mention above, implies at the beginning of the nineties, the appearance of the distributed data processing environment and of the client/server architecture.

Whereas the previous systems were "owner systems", the developments of client/server systems on UNIX allowed to decentralise the processing with a centralised data base and with a relevant level of aggregation of these data.

For instance, in one Industrial project, we have developed a client/server architecture with a Master Production Schedule managed by a micro-computer strong linked with the industrial strategy, with a module of MRP and Purchasing at the middle term level managed by a departmental computer (medium size) (core of the architecture) and finally, with several short term modules at the shop or cell level managing the real time scheduling, as shown below :

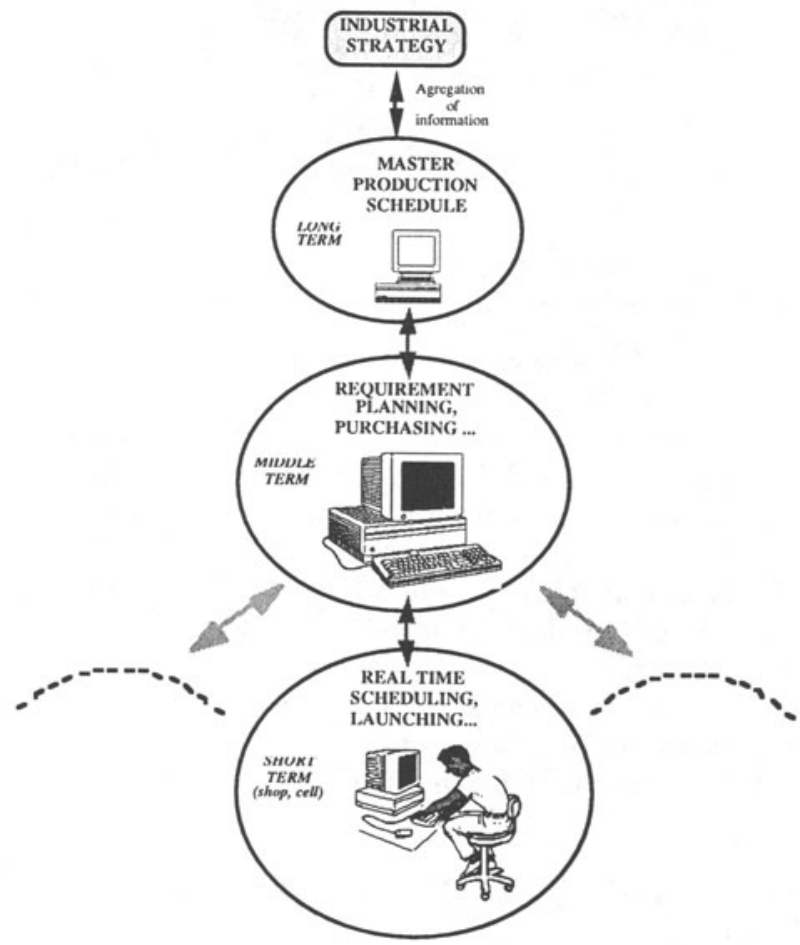

Figure 5 An example of client/server architecture

The main advantage of this system is the modularity. This modularity allows to assure the evolution of the system. This evolution is required to support the flexibility of the production systems, its various changes during the continuous evolution of the 
production system and then to have the most appropriate production management to answer to the customer requirements and to the market evolutions.

The second kind of packages are the Integrated Packages. Today, a new generation of packages are proposed by the main software editors : these are packages which integrate the various functions of the enterprise with the production function. The disadvantage of these packages are the complexity and the difficulties to adapt them to the specificity of each enterprise. Moreover, in this kind of packages, the production management is missed in comparison with other functions as accounting or sales. SAP, BAAN are certainly the most representative of this kind of Packages.

\subsection{Towards the Customisation of Production Management System.}

We have seen the difficulties to choose a PM Package and sometime to implement it. There is today an important debate between two points of view :

- first, some specialists recommend to choose any recognised PM Package (PMP), to implement with a minimum of preparation and then, the PMP will structure the organisation. This point of view is more a IT specialists point of view.

- at the other extremity of this point of view, some specialists (often coming from manufacturing side) recommend to choose carefully the PMP based on an elaborated specifications book. They recommend, then, to adapt both : the organisation and the PMP.

Where is the truth ? As usual between the both points of view. We don't believe it is possible to adapt "drastically" the organisation, but we know also that the PMP adaptation is limited. Anyway, we have to mention the possibility for adaptation of the PMP to use with the Client/Server technology, the potentiality of high level software development tools such as ACCESS or FOXPRO.

In this way, it is possible to create customised applications without incurring the high cost of a long software development.

\subsection{The use of object oriented approach.}

The advantages are as important for the programmer as for the users. In one hand, for the programmer, this approach allows to facilitate the development, to increase the modularity of the package, to facilitate the definition of parameters and then the implementation.

On another hand, for the user, this approach increases the user-friendliness, the adaptability of the production system changes. Indeed, one or several modules can be developed for a same function according to the requirements. It allows to have the most appropriate package for the system. We can see today available software as PROTEAN (MARCAM) for process industry.

\section{CONCLUSION}

Despite the recent evolutions of production management, a lot of work must be still perform, in particular to integrate human and social aspects.

The danger with integrated packages is the lost of flexibility, of understanding, the increasing of complexity and of the difficulties during the implementation due to the 
long time of implementation.

However, the Enterprise Modelling Techniques can improve the situation, by facilitating the implementation of PMP by ,on one side, adapting the organisation and on the other side helping to describe and to understand the PMP.

The development of packages more open, more modular, in particular with the use of object design, will facilitate the implementation.

\section{Acknowledgement:}

The authors want to acknowledge Professor Jim Browne, Professor Eero Eloranta and Professor Asbjorn Rolstadas which have given a strong contribution to the realisation of this paper.

\section{REFERENCES}

Scheer A.W. (1994) "Business Process Engineering : Reference models for industrial enterprises " Springer-Verlag -

Browne J., Harhen J., Shivnan J. (1988) - "Production management systems" Addison Wesley edition - 284p

Doumeingts G., Vallespir B. (1992) - "La gestion de production" - Techniques de l'ingenieur - A 8265 - 24 pages.

Doumeingts G., Breuil D., Pun L. (1983) "La gestion de production assistée par ordinateur" - HERMES.

Doumeingts G., Ducq Y., Clavé F, Malhéné N. (1995) "From CIM to Global Manufacturing" - CAPE 95 - Beijing - China -

Doumeingts G., Ducq Y., Kleinhans S., Clavé F. (1996) "The GRAI Approach to improve the competitiveness of industrial enterprises" - Mannheimer Unternehmerforum - June 27-28th 1996 - Mannheim -

Higgins P., Le Roy P., Tierney L. (1996) "Manufacturing Planning and Control : Beyond MRP II" - Chapman \& Hall - 1996 - 235p -

Vollmann T.E., Berry W.L., Clay Whybark D. (1988) "Manufacturing planning and control systems" - IRWIN -

Williams and all (1994) "Architectures for integrating manufacturing activities and enterprises" - Computer in industry "Special CIM Architectures"- volume 24 number 2-3 - Septembre 1994 - ELSEVIER -

\section{BIOGRAPHY}

Guy Doumeingts is presently Professor at the University of Bordeaux 1, Director of the LAP (Laboratoire d'Automatique et de Productique) and Head of GRAI (Groupe de Recherchen en Automatisation Integree). He graduated from the University of Bordeaux 1 and received his Ph.D. degree from the same University. He is one of the founder of the GRAI Group which is a pioneer in the field of Enterprise Modelling. He has published over 150 articles and three books. He is the Chairman of the Technical Committee No.5 "Computer Application in Technology", of IFIP (International Federation for Information Processing), a Member of SME, AFCET, AFGI. He is a member of the Editorial Board of four International Journals. 